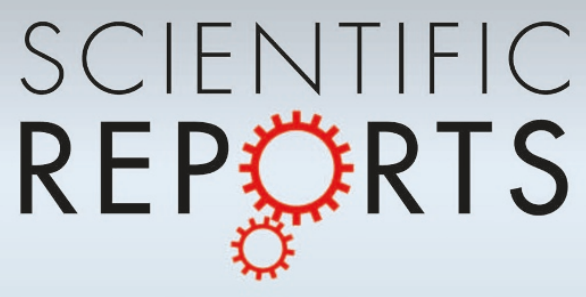

OPEN

SUBJECT AREAS:

RETROVIRUS

ELECTRICAL AND ELECTRONIC ENGINEERING

Received

20 August 2014

Accepted

6 October 2014

Published

30 October 2014

Correspondence and requests for materials should be addressed to M.A.A. (m.alahmad@ vaeu.ac.ae) or F.M. (fmustafa@uaeu.ac.

ae)

\title{
Virus detection and quantification using electrical parameters
}

\author{
Mahmoud Al Ahmad' ${ }^{\text {, Farah Mustafa }}{ }^{2}$, Lizna M. Ali ${ }^{3}$ \& Tahir A. Rizvi ${ }^{3}$
}

\begin{abstract}
'Department of Electrical Engineering, College of Engineering, United Arab Emirates University, Al Ain, UAE, ${ }^{2}$ Department of Biochemistry, College of Medicine and Health Sciences, United Arab Emirates University, Al Ain, UAE, ${ }^{3}$ Department of Microbiology and Immunology, College of Medicine and Health Sciences, United Arab Emirates University, Al Ain, UAE.
\end{abstract}

Here we identify and quantitate two similar viruses, human and feline immunodeficiency viruses (HIV and FIV), suspended in a liquid medium without labeling, using a semiconductor technique. The virus count was estimated by calculating the impurities inside a defined volume by observing the change in electrical parameters. Empirically, the virus count was similar to the absolute value of the ratio of the change of the virus suspension dopant concentration relative to the mock dopant over the change in virus suspension Debye volume relative to mock Debye volume. The virus type was identified by constructing a concentration-mobility relationship which is unique for each kind of virus, allowing for a fast (within minutes) and label-free virus quantification and identification. For validation, the HIV and FIV virus preparations were further quantified by a biochemical technique and the results obtained by both approaches corroborated well. We further demonstrate that the electrical technique could be applied to accurately measure and characterize silica nanoparticles that resemble the virus particles in size. Based on these results, we anticipate our present approach to be a starting point towards establishing the foundation for label-free electrical-based identification and quantification of an unlimited number of viruses and other nano-sized particles.

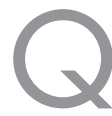

uick label-free virus screening and quantification techniques are becoming increasingly important ${ }^{1}$. Most of the existing techniques suffer from limitations due to the need for extensive sample preparation, or are extremely costly and time consuming ${ }^{2-3}$. A systematic label-free virus quantification and identification by electrical parameters has not yet been defined or developed.

Virus quantification methods based on antigen detection, such as the gag p 24 antigen $^{3-4}$ or genome detection ${ }^{5-8}$ are virus-specific and sometimes suffer from a limited dynamic range of detection. Particularly noticeable are the enzyme-linked immunosorbent assays (ELISA) ${ }^{9-11}$. Quantitative methods such as real time PCR $^{7,8,12}$, viral pla$q^{2 u} e^{13}$ and colony forming (CFU) assays ${ }^{14}$ have been developed to aid the determination of virus titers, while others such as transmission electron microscopy (TEM) ${ }^{15}$ and different mass spectroscopy techniques ${ }^{16}$ have been developed to provide more accurate and reliable tools to measure virus size and charge; however, they are expensive and highly time consuming to perform. Most of these techniques also require high concentrations, which are difficult, if not impossible to achieve during the early diagnosis of many diseases.

This work attempts to explore and demonstrate the use of the semiconductor theory and principles ${ }^{17}$ for virus identification and quantification. The basic premise behind the idea was that application of an electric field should lead to polarization of the virus particles and the medium. Figure 1(a) shows a schematic of the virus particle distribution inside a coaxial adaptor (the measuring chamber) with no applied electric field. With the application of electric field, the particles should be redistributed according to their polarization polarities as presented in Fig. 1(b). The strength of polarization depends upon the composition of the virus itself and its interaction with the polarity of the medium. All viruses are coated with proteins and contain genetic material which can either be DNA or RNA ${ }^{18,19}$. Since both nucleic acids have phosphodiester bonds, the genetic material provides a partial negative charge to the virus ${ }^{18}$. The viral nucleic acid genomes are wrapped in proteins that can be neutral, negative, or positive in charge ${ }^{19}$. Therefore, the net charge of a virus depends upon the cumulative charges of the genetic material and the protein.

\section{Current Approach}

When a voltage is applied to a medium containing a virus suspension, the propagated electric field distribution is altered in magnitude and phase depending upon the virus content and its relative concentration which is considered an "inhomogeneity". The virus particle could be present as an electrical dipole which has two pairs 
(a)

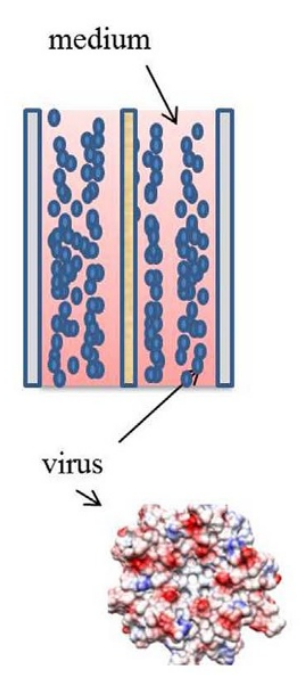

(b)

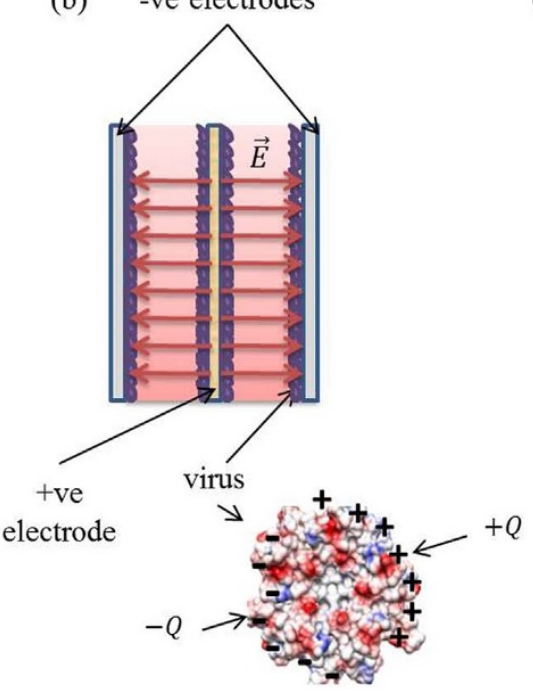

(c)

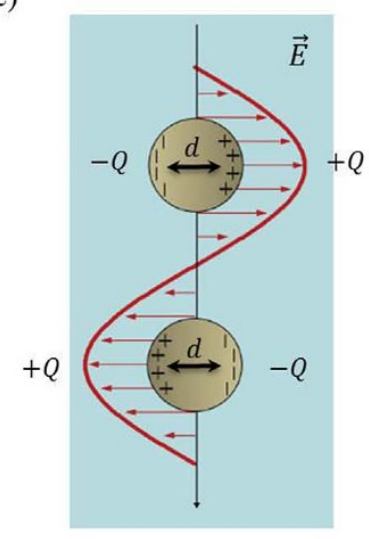

Figure 1 Illustration of the rationale of the electrical measurement for virus titer measurement and classification. (a) Virions distributions inside the coaxial resonator in the absence of electric field. (b) Polarized virions when an electrostatic field is applied. A coaxial resonator has an inner conductor (the + ve electrode, where positive charges accumulate, $+Q$ ) surrounded by a hollow space that is surrounded by a conducting shield $(-$ ve electrode, where the negative charges accumulate, $-Q$ ). (c) Schematic of the polarized virus particles inside an alternating current electric field.

of electrical charges of equal magnitude but opposite sign, separated by some distance $(d)$, as depicted in Fig. 1(c). It is, therefore, suggested to consider the virus particles as "impurities" (dopant) that exist in non-intrinsic semiconductor materials.

The principle of the present approach is to calculate the doping concentration of the suspension and de-embed the contribution of the reference medium. Although the ratio of the virus inside the medium is of a small percentage, it still produces a dramatic change in its electrical properties, depending upon the accumulated charge of the virus.

The particle count of the suspension $(\vartheta)$ empirically is found to be the absolute value of the ratio of the differences between the virus suspension dopant $\left(N_{S}\right)$ and mock medium dopant $\left(N_{M}\right)$ divided by the difference between the suspension Debye volume $\left(D_{S}\right)$ and the medium Debye volume $\left(D_{M}\right)$. Mathematically, the particle count thus can be represented as:

$$
\vartheta=\left|\frac{N_{S}-N_{M}}{D_{S}-D_{M}}\right|
$$

The suspension and medium doping concentrations can be extracted from the capacitance-voltage $(\mathrm{CV})$ measurements using the following equation ${ }^{17}$ :

$$
N=\left|\left(0.5 q \varepsilon A^{2} \frac{d\left(1 / c^{2}\right)}{d V}\right)^{-1}\right|
$$

where: $q, \varepsilon, A, C$ and $V$ are the electron charge, dielectric constant of the effective reference medium (or the effective suspension), overlapping area, measured capacitance and applied bias, respectively.

In terms of identification, the type of virus was identified based on a set of electrical parameters which were extracted from the corresponding current-voltage (IV) and capacitance-voltage (CV) profiles. As in semiconductor theory, a new parameter was defined, $\alpha$, that connects the virus concentration with its relative change in mobility to identify the virus type. Hence, the virus form can be identified using the following parameter:

$$
\propto=\left|N_{S}-N_{M}\right| /\left|\mu_{S}-\mu_{M}\right|
$$

where $\mu_{S}$ and $\mu_{M}$ are the extracted mobilities of virus suspension and mock medium, respectively. As will be seen, this parameter became a unique identifier for each virus type and was used for detection purposes.

\section{Results and Discussion}

To establish the proof-of-principle, two closely related viruses were analyzed in this study: feline immunodeficiency virus (FIV) and human immunodeficiency virus (HIV). These two viruses belong to the lentivirus group of retroviruses and are of close morphology and dimension $(\sim 110-150 \mathrm{~nm})^{20}$. The main reason for choosing two similar viruses with almost same morphology and size was to demonstrate the sensitivity of the current method. The two different virus samples were prepared using transfection techniques as reported earlier, employing a calcium phosphate transfection protocol (see Methods) $)^{21,22}$. To ensure that the medium in which the virus was released by the virus-producing cells was free of any cellular debris, the low-conductive tissue culture medium was first centrifuged at low speed and the clarified medium was filtered through a 0.2 micron filter, ensuring that only the virus particles remained in the media which was composed of Dulbecco's Modified Eagle Medium (DMEM) supplemented with the required fetal bovine serum and appropriate antibiotics. To perform the experiment, the virus suspensions were individually loaded inside the open-ended coaxial cable and the data gathering was achieved by using the Gamry 3000 equipment (USA) that has a wide range of electrical measurement capability.

Figure 2 shows the electrical behavior (polarization capability) of FIV and HIV partciles along with the mock medium (medium without virus) when subjected to an electric field. The mock medium went through all the steps of transfection that the test samples went through to ensure that it had the same $\mathrm{pH}$ and polarity as the virus preparations. As can be observed, the charging profile of the mock medium was nearly constant (Fig. 2(a)), which implies that the mock exhibits a linear response. Charging of the virus particles was not instantaneous and took a finite amount of time in the order of a few seconds to accumulate. Considering the virus-free mock medium as a reference, the charging of the FIV particles was faster than HIV which needed more time to get charged. On the other hand, the discharging profiles (Fig. 2(b)) revealed that the HIV particles discharged faster than FIV. The discharging of the virus particle occurs due to the redistribution of charges bound within the virus particles under the influence of an applied electric field during the polarization 
(a)

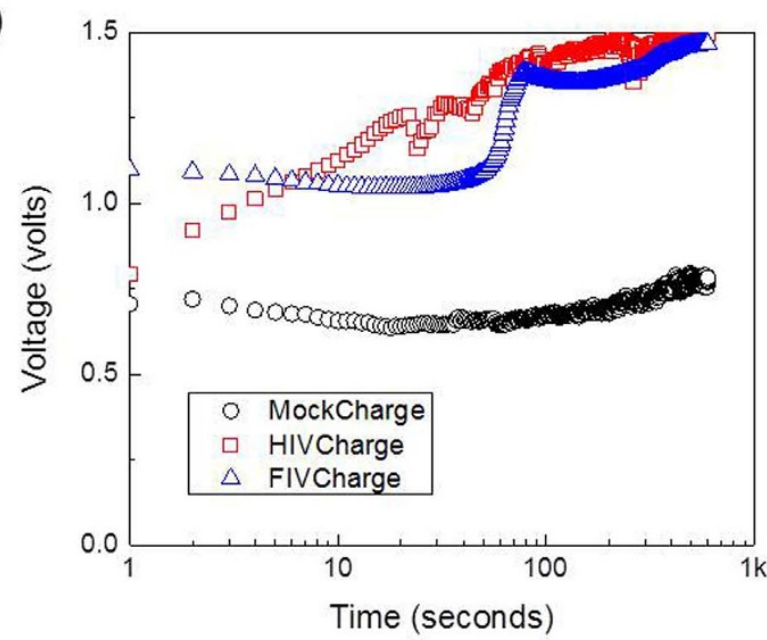

(c)

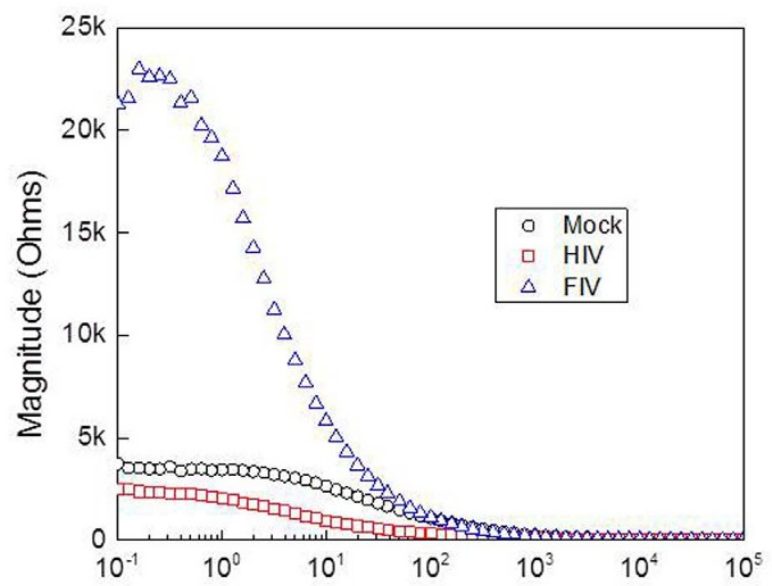

(e)

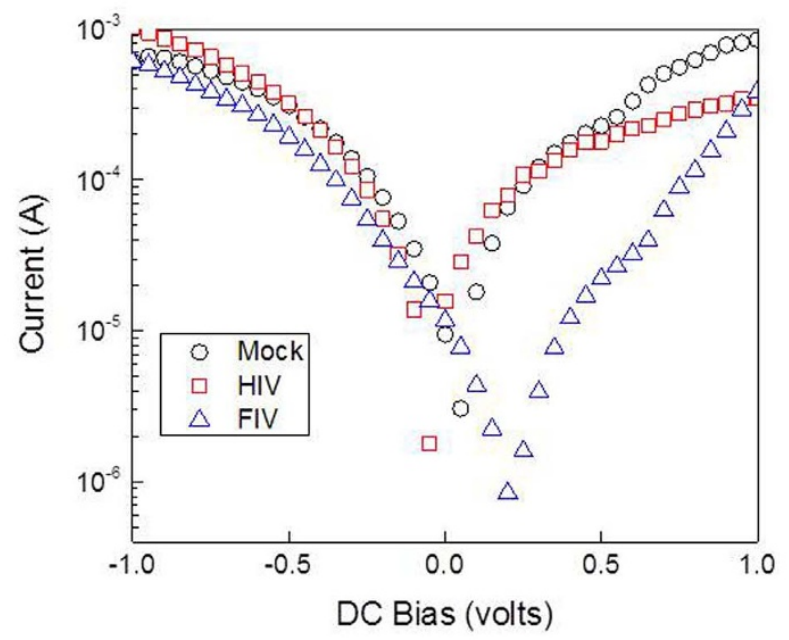

(b)

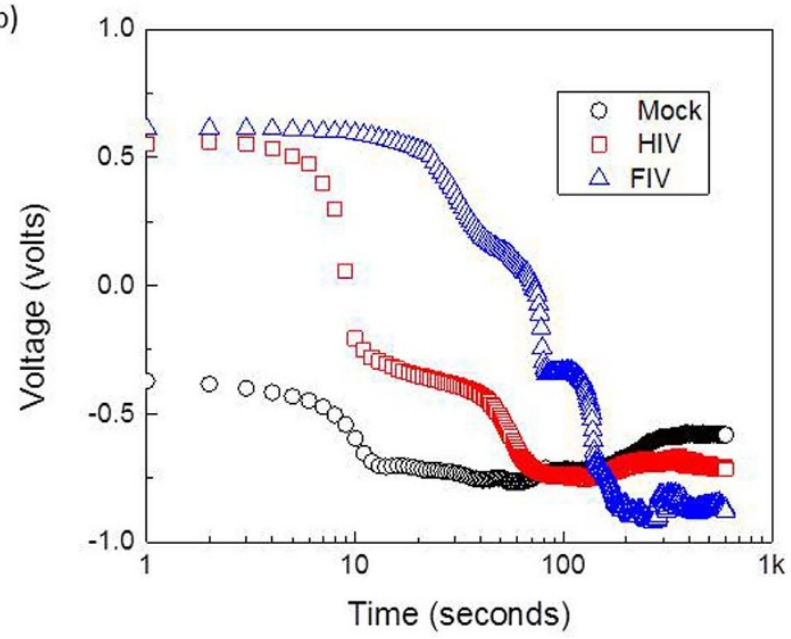

(d)
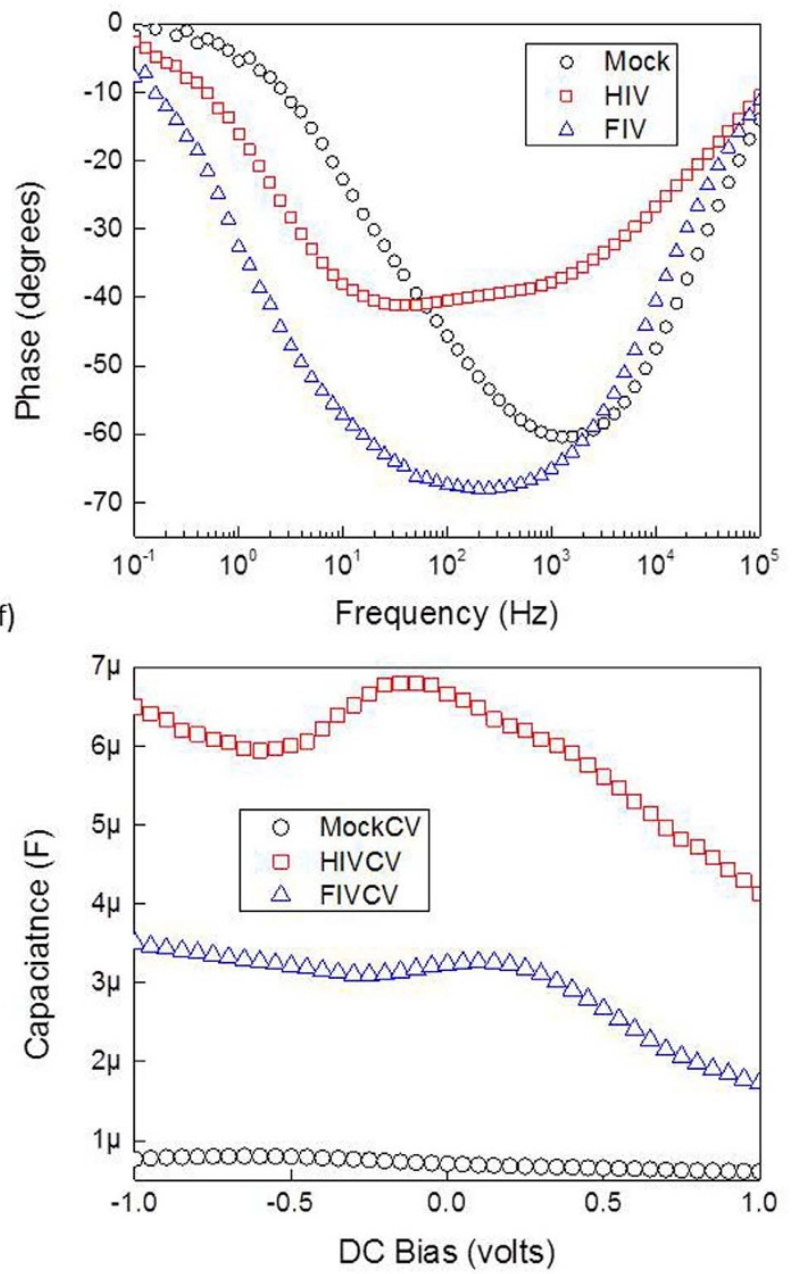

Figure 2 Electrical measurements: (a) charging and (b) discharging curves for mock (virus-free media), FIV and HIV particles. The measurements were conducted for up to 15 minutes at constant capacity of $1 \mathrm{~mA}-\mathrm{hr}$. The charging/discharging currents were kept at the constant value of $0.5 \mathrm{~mA}$ and were conducted over 15 minutes as well. (c) Impedance magnitude and (d) phase measurements versus frequency of virus particles compared to the mock. (e) Current-voltage (IV) and (f) Capacitance-voltage (CV) measurements versus bias profiles.

process, whereby charges of the opposite polarity and same magnitude are displaced to different locations within the suspension. When the applied electric field was removed, the suspension returned to its original state within a finite time interval needed by the charge equilibrium to be established in the suspension. As the suspension mixture acts as a dielectric material, the presence of virus particles stabilized the charging profile due to their ability to store the electrical charges. Thus, the change in the corresponding electrical responses of the viruses was due to their associated intrinsic properties. 
It is well known that the charge of genetic material and proteins can be largely influenced by the complex composition of the liquid suspension medium, such as the $\mathrm{pH}$, ion strength, and any inorganic and organic substrates ${ }^{19}$. To normalize for such influences, to reiterate, our technique incorporated a de-embedding step where such changes were automatically considered by default by utilizing the mock medium as a reference. This medium sample underwent all the procedures that the test samples had undergone. Therefore, any relative changes in the $\mathrm{pH}$, composition, and polarity of the virus suspension were de-embedded using the mock values in the proposed technique, validating the experimental and theoretical analyses.

Figure 2(c) \& (d) demonstrate the impedance magnitude and phase measurements, respectively. When compared to the mock sample, the corresponding HIV impedance magnitude decreased, exhibiting a negative differential resistance, while the FIV particles introduced further electrical losses as its impedance magnitude increased. On the other hand, when compared to the mock phase, the phase of the FIV particles increased, while the phase of the HIV particles decreased (Fig. 2 (d)). These observations suggest that the effective dielectric constant of HIV is higher than FIV. Thus, the effective constant value depends on different parameters namely, size, concentration, volume and composition.

Next, the IV and CV measurements were conducted at mid-band frequency of the capacitive region to ensure a capacitive behavior (Figure 2(e) \& (f)). The IV demonstrated a schottky-like barrier junction (a potential energy barrier for electrons formed at the metal-semiconductor junction) within which the "turn on" voltage is highly dependent on the intrinsic property of the suspended medium inside the coaxial adaptor. Thus, the "turn on" voltage strongly depended on the virus load and its type: whether it introduces losses (as FIV) or exhibits a negative differential resistor (as was the case with HIV). The CV profiles were plotted in Figure 2(f) and showed a smooth capacitance-voltage dependency. The effective dielectric constants of HIV and FIV particles were higher than the mock constant by 9.5 - and 4.5 -folds, respectively. This difference strongly depended upon the volume of the suspended viruses as well as their electrical properties. As the results show, the viruses exhibited a semiconducting behaviour which could be used to quantify the virus particles within the medium.

Next, the electrical "signatures" of the viruses were extrapolated from the results obtained as follows: when the virus particles are more polarisable than the suspension medium, the charge density at the internal aspect of the interface between the particles and the medium is greater than the charge density at the external aspect of the interface. Thus, the net induced dipole on the virus particle is aligned with the applied electric field. The electric field lines then bend towards the virus particle. When the virus particles are less polarisable than the medium, the charge density at the internal aspect of the interface between the particle and the suspending medium is less than the external aspect of the interface. Thus, the net induced dipole aligns counter to the applied electric field. The electric field lines should bend around the virus particles as if they were an insulator. When the charge density at the internal aspect of the interface between the virus particle and the mock is equal to the external aspect of the interface, the polarisability of the virus particle and mock medium should be equal and the applied electric field should remain unperturbed. Such a strategy streamlined the interpretation of our results in terms of specific electrical "signatures" for these two different viruses, which could then be attributed solely to the specific nature of respective viral particles since the media in which they were suspended in was the same.

Applying the technique and the presented theory in the section of electrical parameters extraction algorithm, the set of corresponding extracted electrical parameters of the mock and suspended mixtures are listed in Table 1. Each column represents the corresponding values for each material type. The values strongly depended upon the concentration of the suspensions and any other possible interactions between the particles and their relative medium. The extracted doping of the impurities, their mobilities, and Debye length were used to compute the concentrations and classifications values. As noticed, the impurities (doping) were inversely related to the Debye length and depletion width.

Comparison of the Debye length of the mock medium with that of the HIV and FIV particles revealed that the length decreased and increased, respectively, depending upon the nature of the virus particle. This can be explained as follows: the mock material intrinsically exhibits a semiconducting junction within which the electrons and holes (position previously occupied by the electron) are attracted to the sides of the used coaxial structure relative to the applied DC bias, as depicted in Fig. 3(a). With bias, the FIV particles get charged with the same polarity as the mock material, thus increasing the depletion width, whereas the HIV particles charge with an opposite polarity of the mock material, resulting in depletion-width reduction. Thus, the HIV particles have enhanced the forward bias characteristics, while the FIV particles have enhanced the reverse bias characteristics, despite the fact that biologically they are highly related viral species.

To confirm that the quantification and classification of the viruses was unique and due to their intrinsic properties, silica nanoparticles (obtained from Nanocomposix, USA) with a diameter of $120 \mathrm{~nm}$ and adjusted to a concentration to $2.4-5.4 \times 10^{11}$ were suspended in water and their suspension concentrations were measured using the more classical approaches of the Agilent 8453 ultraviolet/visible spectrophotometry (UVIS). Their measured concentration distribution versus nanoparticle size is depicted in Fig. 3(b). The reason for including silica nanoparticles with similar size and concentration was to demonstrate the selectivity of the proposed technique. The corresponding IV and CV measurements of the nanoparticle suspension and their blank water medium were performed and are depicted in Fig. 3(c) and (d), respectively. Similar to the viruses, the IV profile of the nanoparticles also exhibited a schottky-like diode property typical of a semiconductor material (low voltage drop

\begin{tabular}{|c|c|c|c|c|c|c|}
\hline Parameter & Description & Mock & HIV & FIV & Water & Silica \\
\hline$\sigma$ & Conductivity $\left[\times 10^{-5} \Omega^{-1}\right]$ & 30.66 & 35.5 & 11.9 & 18.8 & 10.3 \\
\hline$L_{D}$ & Debye length $[\mu \mathrm{m}]$ & 59 & 34 & 125 & 44 & 101 \\
\hline$W_{D}$ & Depletion width $[\mu \mathrm{m}]$ & 318 & 186 & 719 & 247 & 552 \\
\hline$\mu$ & Mobility $\left[\times 10^{13} \mathrm{~cm}^{2} /(\mathrm{V} \cdot \mathrm{s})\right]$ & 7.6546 & 52.431 & 1.1305 & 0.078 & 0.045 \\
\hline$\vartheta$ & Concentration $[\# / \mathrm{mL}]$ & NA & $4.42 \times 10^{11}$ & $6.59 \times 10^{11}$ & NA & $3.83 \times 10^{11}$ \\
\hline$\alpha$ & Classification $\left[\times 10^{-4}\right]$ & NA & 9.87 & 18 & NA & 6279 \\
\hline
\end{tabular}


(a)

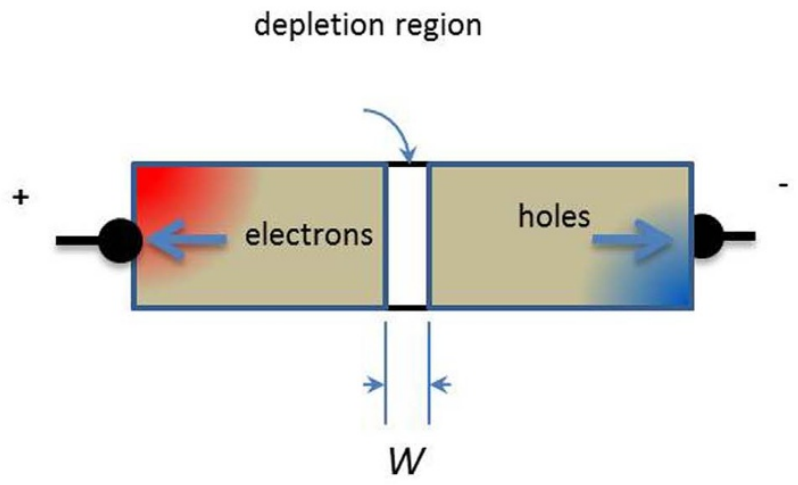

(c)

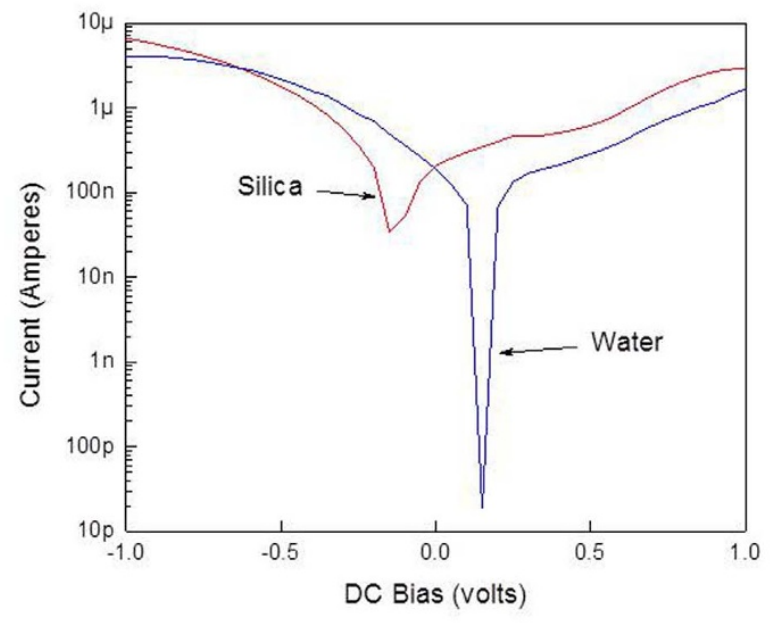

(e)

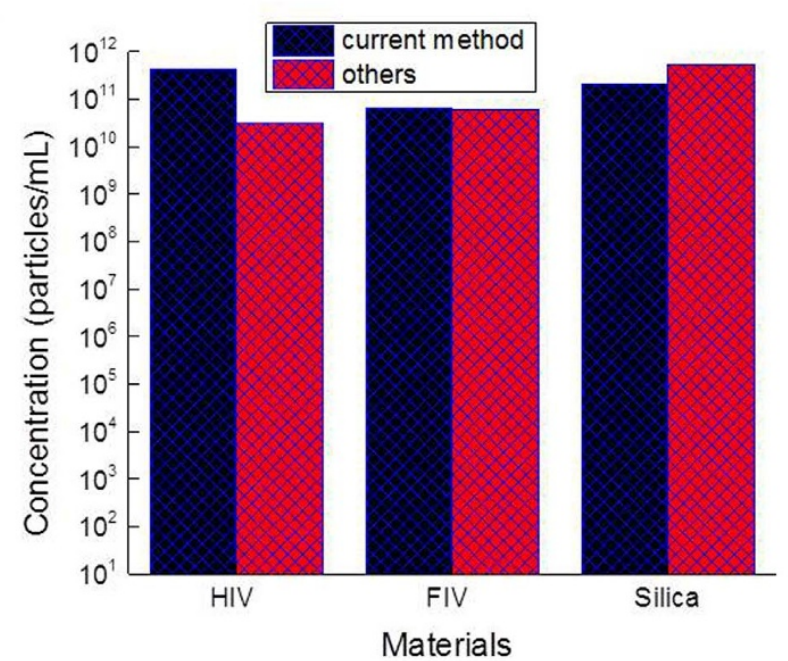

(b)

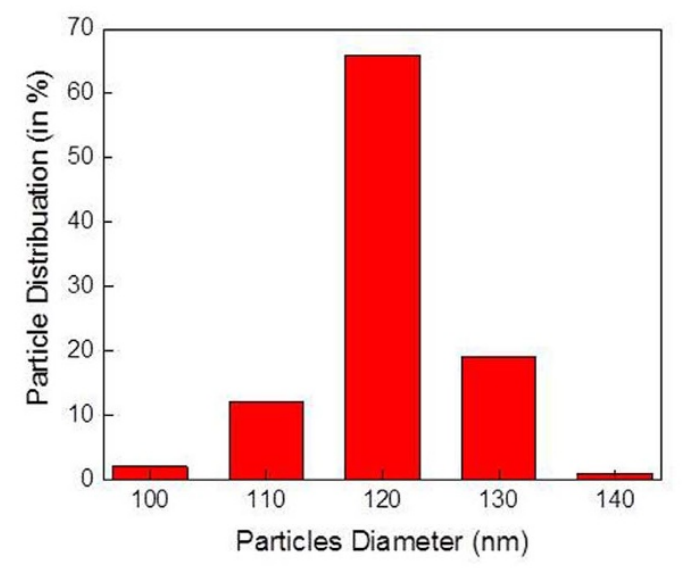

(d)

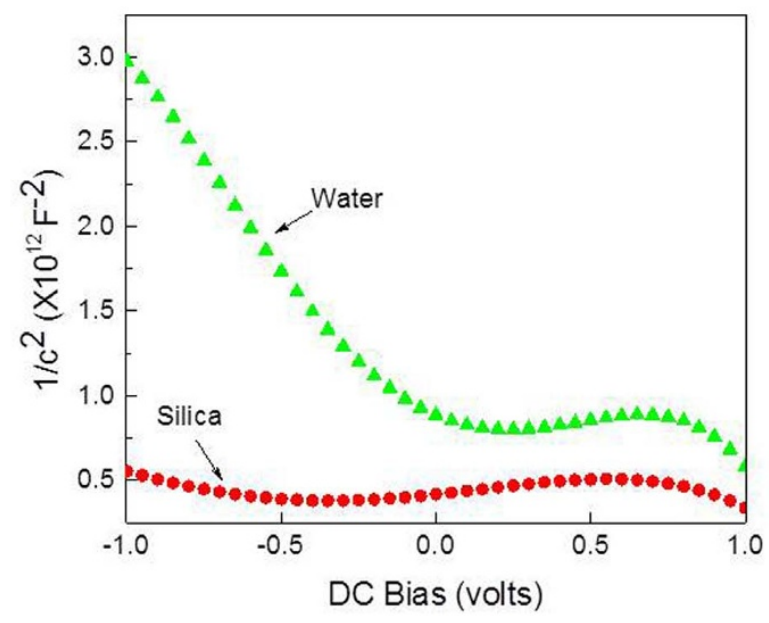

(f)

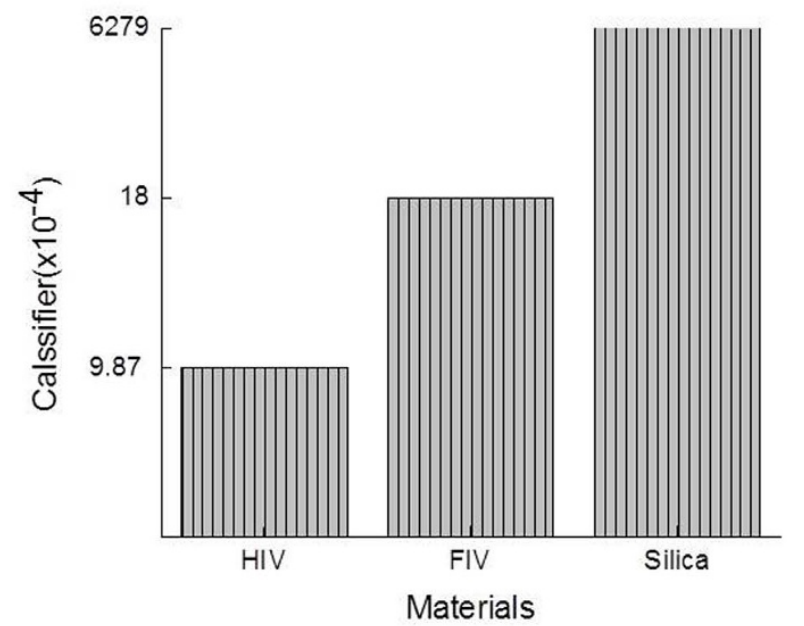

Figure 3 Quantification and characterization of silica nanoparticles, HIV, and FIV particles: (a) Schematic representation of the semiconducting junction. "Holes" refer to the "vacant" position occupied by the moving electrons in the semiconducting material. W, width of the "depleted region". (b) Relative percentage of the particle distribution over diameter using the UVIS technique. (c) Current-voltage and (d) Capacitance-voltage measurements of silica nanoparticles using the electrical technique. (e) Comparison of concentrations obtained with the electrical method compared to other conventionally-used techniques relevant to the material tested. The concentration of the viral particles (VP) per milliliter (ml) was found to be as follows: HIV $=2.0-3.2 \times 10^{10} \mathrm{VP} / \mathrm{ml}$, FIV $=3.6-6.2 \times 10^{10} \mathrm{VP} / \mathrm{ml}$. The silica nanoparticle concentration was measured to be $2.4-5.4 \times 10^{11}$ per $\mathrm{ml}$. (f) The classifier parameter (signature value) for the different materials used. 
and fast switching action). Based on these measurements, the extracted particle concentration using our proposed technique was found to be $3.83 \times 10^{11}$ particles/ml; i.e., within 1.5 folds of the expected nanoparticle load (Table 1).

Similarly, electrical measurements were used to estimate the HIV and FIV counts which were measured to be $4.42 \times 10^{11}$ and $6.59 \times$ $10^{11}$ particles per $\mathrm{ml}$, respectively, as listed in Table 1 . Test of the same viral supernatants for virus particle quantitation using the QuickTiter $^{\mathrm{TM}}$ Lentivirus Quantitation Kit (Cell Biolabs Inc., San Diego, CA USA) revealed virus titers of 2.0-3.2 $\times 10^{10}$ and 3.6-6.2 $\times 10^{10}$ for HIV and FIV, respectively. This kit allows one to measure both the HIV and FIV virus particles using the same method (nucleic acid quantification) rather than two different virus-specific assays (such as ELISAs or real time assays) that would have had variable sensitivity and specificity against each viral species, resulting in particle numbers that could not have been compared with each other.

Results from these two vastly different techniques (electrical verses biochemical) were within one order of magnitude, demonstrating the validity of the proposed electrical technique for virus quantitation. The slightly lower estimates of virus particles using the biochemical technique compared to the electrical technique for the two viruses may reflect the inability of this assay to detect any "ghost" viral particles that may have formed devoid of any nucleic acid ${ }^{23}$.

Figure 3(e) summarizes the data obtained for the different types of materials used in this study (FIV, HIV and silica nanoparticles), their extracted concentration amounts using the current electrical method, along with their corresponding values obtained from other established methods used to quantify them. Taken together, the data presented here suggests that there was strong concordance between the electrical method and the other methods used to estimate particle concentration, whether the particles were biological entities (viruses) or inert in nature (silica nanoparticles). Furthermore, comparison of the "classification" parameters of the three species (HIV, FIV, and silica nanoparticles) revealed that each entity had its own electrical "signature" whether they were closely related (such as HIV and FIV) or different (such as the nanoparticles) (Figure 3(f)). These observations strongly suggest that this parameter could be used to characterize and identify each "impurity" introduced into the semiconductor material (mock media or water). Here, we would like to emphasize that in case of the complex biological media, the method could still detect the electrical signature of the virus. This is because no matter what the solution to suspend the virus in, the electrical signal from the solution is "de-embedded" from the final signal

(a)

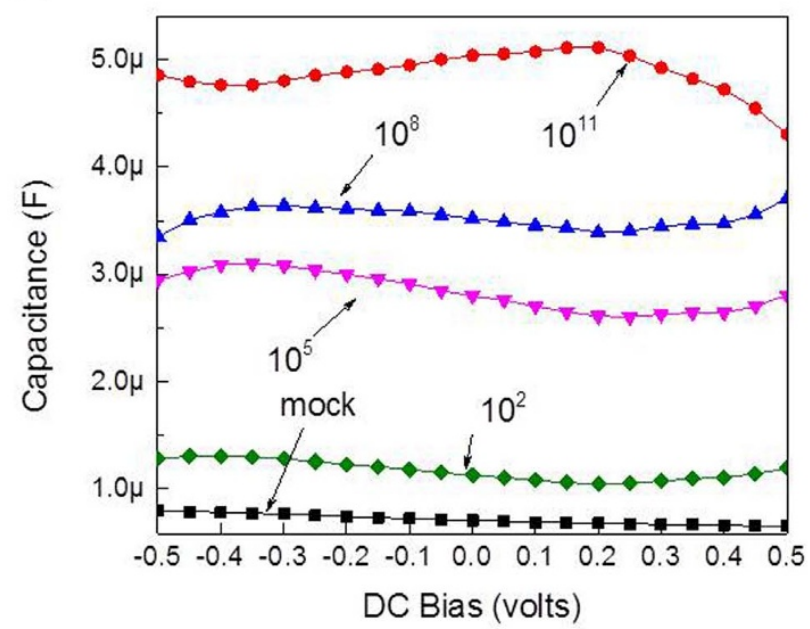

obtained with the virus suspension. That is why the suspension solution is always used as a control. Thus, the electrical signal detection is conducted "irrespective" of the media in which the virus is suspended in.

Finally, the sensitivity of the electrical method was tested by diluting a known concentration of FIV with its relative blank reference medium. As shown in Fig. 4(a), the more concentrated the virus particles, the higher the observed effective capacitance value. The change in capacitance as a function of the extracted virus concentrations is depicted in Fig. 4(b) which showed a linear relationship, revealing the accuracy of the estimation. The technique described here was observed to be sufficiently sensitive to allow a limit of detection of several hundred FIV virus particle $/ \mathrm{ml}$, which is considerably more sensitive than most of the current methods available given the fact that no sample preparation or labeling was required to make the estimates and the process took only less than a minute.

\section{Accuracy and reproducibility of the electrical method}

The accuracy and reproducibility of the presented method has been checked using repeated electrical measurements against multiple virus stocks prepared at different times; i.e., all within the employed frequency range and over the same applied bias voltage. As detailed, the accuracy of these measurements using the outlined methodology is comparable with other conventional techniques.

\section{Conclusion}

In summary, the methodology presented here is a basic attempt to demonstrate and explore the use of a new technique for virus detection, classification and identification. The outlined technique should be applicable to any type of virus, provided it can be electrically polarized, as well as other types of particles in suspended solutions. In addition, the proposed methodology provides a better combination of high sensitivity, selectivity, quick response, low cost, high throughput, and ease of use without the need of any biomarker or labelling techniques. When compared with other conventional identification and quantification techniques, this approach was found to be the fastest (within minutes) and cheaper than any other known technique. Finally, the proposed technique can be upgraded to be applied in situ, which will not only pave the way for direct and rapid detection of viruses in biological samples, but also enable their early stage detection in infected individuals.

\section{(b)}

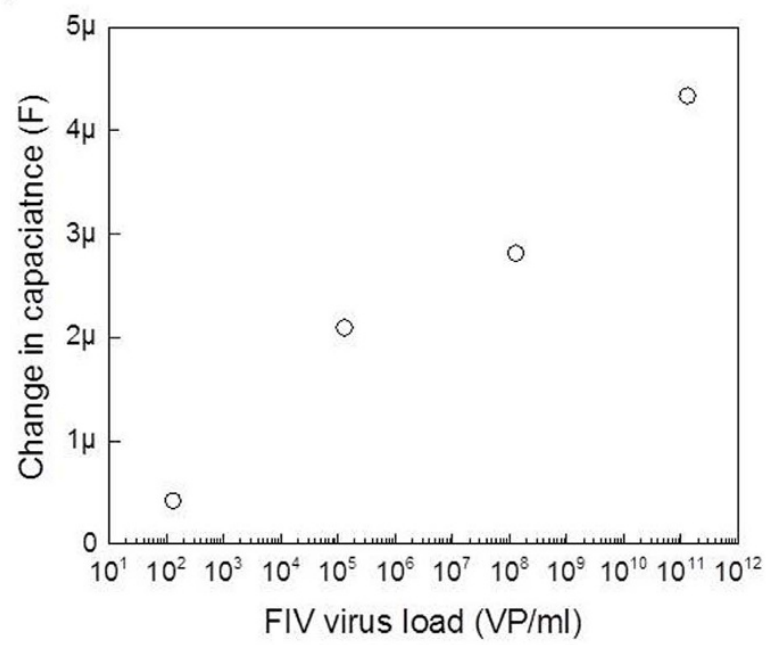

Figure 4 Sensitivity assessment of the electrical method for virus quantitation: (a) measured capacitance-voltage dependency for different dilutions of FIV particles, and (b) change in capacitance versus the extracted FIV load. 


\section{Methods}

Electrical characterization methods. The whole set of electrical measurements were carried out using the Gamry Reference 3000 instrument (Gamry/USA). The Reference 3000 is a high-current, high-performance USB potentiostat with 11 current ranges from 3 amps to 300 picoamps and compliance voltage up to 32 volts. With onboard electronics for electrochemical impedance spectroscopy, the Reference 3000 can make measurements over a frequency range from $10 \mu \mathrm{Hz}$ to $1 \mathrm{MHz}$. The impedance measurements were performed in the range $100 \mathrm{mHz}$ to $100 \mathrm{KHz}$ at an applied oscillation voltage of $15 \mathrm{mVpp}$. The selection of this frequency range was based on earlier measurements at high frequency from $1 \mathrm{MHz}$ up to $13.5 \mathrm{GHz}$ for different types of viruses, namely FIV and HIV. The range $100 \mathrm{mHz}$ to $100 \mathrm{KHz}$ revealed a better identification record of different virus frequency responses, making it easier to identify and characterize the virus using its specific "signature" and also investigate its feasibility for titer quantitation using the present approach. The Reference 3000 involves radio frequency generators that produce power signals at different frequencies, and is capable of measuring current voltage, capacitance voltage, polarization as well as charging/discharging profiles with the ability to change different parameters. The system was calibrated using the manufacture-provided calibration kit. A typical calibration moves the measurement reference plane to the end of the test cables. Therefore, it excludes the effect of losses and phase shifts that could add noise to the measured signal. Each suspension was loaded inside an openended coaxial cable and exposed to the different set of electrical measurements. The self-resonance frequency of the coaxial cables was ensured to be above $100 \mathrm{MHz}$; therefore, it did not affect the measurements in the afore-mentioned range.

Electrical parameters extraction algorithm. With the assumption that virus particles are modeled as impurities in a semiconducting material, different virus load suspensions can be tested for their electrical behavior when charged. The extraction of the semiconductor parameter set associated with each measurement can then be carried out as follows: the leakage current $\left(I_{0}\right)$ and conductivity $(\sigma)$ are extracted from the IV measurements by fitting the forward characteristic with a polynomial represented by equation (4), while the ideality parameter $(n)$ is determined by fitting the IV measurements with the exponential represented by equation (5), respectively. Thus, the mobility $(\mu)$ is then determined using equation (6) below:

$$
\begin{gathered}
I=I_{0}+\sigma V+k V^{2}+\gamma V^{3} \\
I=I_{S} S^{\frac{q V}{n K T}} \\
\mu=\frac{\sigma}{n q}
\end{gathered}
$$

where $I_{0}$ is the leakage current, $I_{\mathrm{S}}$ is the saturation current, $K$ is Boltzmann constant of $1.38 \times 10^{-23} \mathrm{~J} / \mathrm{K}, T$ is the test temperature of $300 \mathrm{~K}$, and $q$ is the electron charge of 1.60 $\times 10^{-19} \mathrm{C} . k$ and $\gamma$ are voltage coefficients.

From the CV experimental measurements, the data can be used to plot $1 / \mathrm{C}^{2}$ verses voltage by taking the slope of the linear part of this curve and with the help of equations (7), (8) and (9) below, the doping concentration, and (N), the dielectric constant of the mock material $\left(\varepsilon_{s}\right)$ can then be computed as follows:

$$
\begin{gathered}
N=\mid 2 /\left(q \varepsilon_{S} A^{2}(\text { slope })\right) \mid \\
C=2 \pi \varepsilon_{r} \varepsilon_{0} l / \ln (b / a) \\
\varepsilon_{s}=\varepsilon_{r} \varepsilon_{0}
\end{gathered}
$$

where $A$ is the capacitor area $\left(94.2478 \mathrm{~mm}^{2}\right)$ and $C$ is the represented coaxial cable capacitance. $\varepsilon_{0}$ is the vacuum dielectric permittivity $\left(8.85 \times 10^{-12} \mathrm{~F} / \mathrm{m}\right), \varepsilon_{\mathrm{r}}$ is the effective permittivity of the mock media, $l$ is the cable length, $b$ and $a$ are the inner and outer radius, respectively. Next, the Debye Length $\left(L_{D}\right)$, bulk potential $(\varphi)$ and the depletion width $(W)$ can be computed using the equations (10), (11) and (12), respectively.

$$
\begin{gathered}
L_{D}=\sqrt{\varepsilon_{s} K T / q^{2} N} \\
\phi=K T \ln (N / \eta) / q \\
W=\sqrt{4 \varepsilon_{s} \phi /} N q
\end{gathered}
$$

Virus culture, sample preparation and biochemical virus quantitation test. The retroviral particles were produced employing the principle of genetic trans complementation used earlier in our laboratory for studying FIV and HIV replication ${ }^{21,22}$. These assays utilize three individual expression plasmids to produce different parts of the virus, including a packaging construct that expresses the viral $\mathrm{gag} / \mathrm{pol}$ structural genes (MB22 for FIV and CMV $\Delta$ R8.2 for HIV-1). These proteins create the retroviral particles which are capable of encapsulating respective retroviral

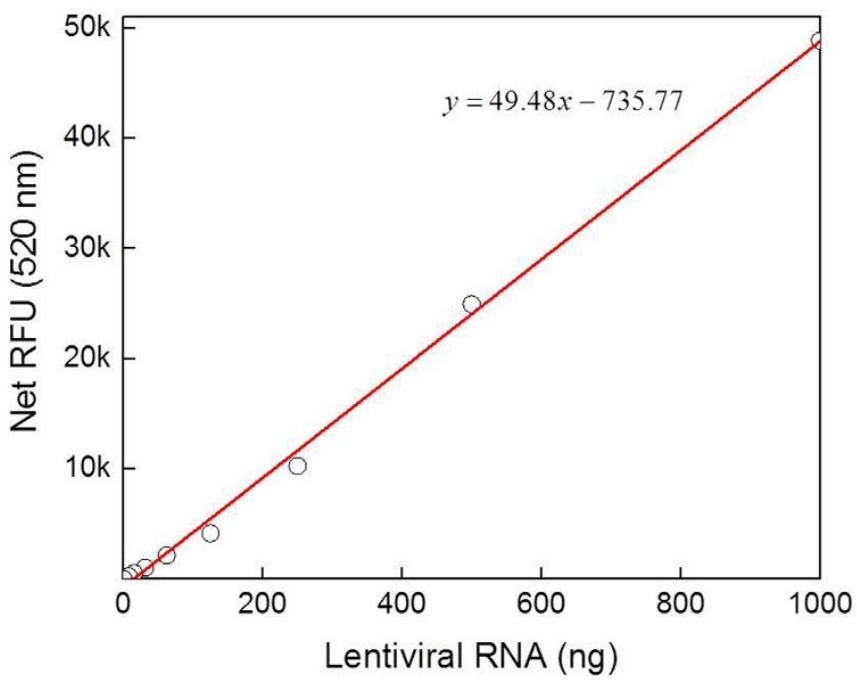

Figure $5 \mid$ A typical RNA standard curve obtained using the pure RNA standards provided in the QuickTiter ${ }^{\mathrm{TM}}$ Lentivirus Quantitation Kit.

RNAs expressed from the packageable RNA provided by the plasmids, TR394 (FIV) and MB58 (HIV-1). These transfer vectors carry the hygromycin resistance marker gene cassette that allows monitoring of successful retroviral infectious events carried out by the virus particles thus produced. The number of hygromycin resistant $\left(\mathrm{Hyg}^{\mathrm{r}}\right)$ colonies obtained thus are directly proportional to the amount of infectious viral particles produced. An envelope expression plasmid (MD.G) based on vesicular stomatitis virus envelope G (VSV-G) was used to pseudotype the two different retroviral particles. The rationale behind pseudotyping different retroviral particles by a common VSV envelope glycoprotein (Env-gp) was that both of these retroviral particles (FIV and HIV) would then be decorated by similar Env-gp.

The three plasmids for constructing each virus (packaging construct + transfer vector + Env-expression construct) were co-transfected into 293T producer cells, which generated the virus particles containing the hygromycin resistance gene cassette. The virus particles were released by the 293T cells into the tissue culture medium used to culture the cells: Dulbecco's modified Eagle medium (DMEM) supplemented with $10 \%$ fetal bovine serum (FBS), penicillin, streptomycin, and gentamycin antibiotics. These virus particles were then used to monitor their electrical signatures.

The QuickTiter ${ }^{\mathrm{TM}}$ Lentivirus Quantitation Kit (Cell Biolabs Inc., San Diego, CA USA) was used to determine the number of FIV and HIV lentiviral particles in the tissue culture media according the manufacturer's directions (Fig. 5). This kit allowed us to measure both the FIV and HIV virus particles using the same assay (based on their nucleic acid content) rather than using two different virus-specific assays that would have had different sensitivity and specificity against each viral species. Briefly, the harvested viral supernatants from the cells were first clarified under a low speed centrifugation step $\left(300 \times \mathrm{g}\right.$ for 5 minutes at $\left.4{ }^{\circ} \mathrm{C}\right)$ and filtered through a 0.2 micron filter to remove any cellular debris. Next, the mock supernatants without virus or with either FIV or HIV were digested with nucleases to remove any source of nucleic acid released from ruptured cells or virus in the supernatant. The viral particles in the mock or test supernatants were captured using proprietary beads in the kit that allowed the specific capture of lentiviral particles. The beads were washed two times in the wash buffer provided and the virus was denatured on the beads, releasing the RNA from the captured viral particles. The RNA released was then detected using a CyQuant ${ }^{\circledR}$ GR RNA binding dye using the Perkin Elmer VICTOR ${ }^{\mathrm{TM}}$ X3 Multilabel Plate Reader using a 485/538 nm filter set. The viral RNA was quantitated against an RNA standard that was provided in the kit for the quantification purposes which gave a linear curve over 1-1000 ng of lentiviral RNA, as shown in Fig. 5.

Since this assay has the capability of detecting $1 \times 10^{10}$ virus particles per milliliter (a medium to high titer range), to ensure that we were within the linear range of detection, a serial dilution of the virus supernatants was tested by this assay in two independent experiments to estimate the final values. Viral titers (virus particles $/ \mathrm{ml}$ ) were calculated as described in the kit using the measured viral gRNA amounts by employing the following formula:

$1 \mathrm{ng}$ lentiviral RNA $=\left(1 \times 10^{-9}\right) \mathrm{g} /(\mathrm{gRNA} M W \times 660 \mathrm{~g} / \mathrm{bp}) \mathrm{X} 6 \times 10^{23}=\mathrm{x}$ virus particles (VP)

Virus Titer $(\mathrm{VP} / \mathrm{mL})=$ Amount of lentiviral RNA $(\mathrm{ng}) \mathrm{X} \times \mathrm{VPX}(20 \mu \mathrm{L} / 5 \mu \mathrm{L}) /$ Sample volume $(\mathrm{mL})$

1. Patolsky, F. et al. Electrical detection of single viruses. Proc. Natl. Acad.t Sci. (USA) 101, 14017-14022 (2004)

2. Vermeire, J. et al. Quantification of reverse transcriptase activity by real-time PCR as a fast and accurate method for titration of HIV, lenti- and retroviral vectors. PLOS ONE 7, 1-12 (2012). 
3. Dong, H. et al. Two types of nanoparticle-based bio-barcode amplification assays to detect HIV-1 p24 antigen. J. Virol. 9, 1-7 (2012).

4. Kim, E. Y. et al. Detection of HIV-1 p24 Gag in plasma by a nanoparticle-based bio-barcode-amplification method. Nanomed. 3, 293-303 (2008).

5. Cotton, M. et al. Full genome virus detection in fecal samples using sensitive nucleic acid preparation, deep sequencing, and a novel iterative sequence classification algorithm. PLOS ONE 9, 1-15 (2014).

6. Baas, C., Barr, I. G., Fouchier, R. A., Kelso, A. \& Hurt, A. C. A comparison of rapid point-of-care tests for the detection of avian influenza A(H7N9) virus, 2013. Euro Surveill. 18, pii, 20487 (2013).

7. Elnifro, E. M., Ashshi, A. M., Cooper, R. J. \& Klapper, P. E. Multiplex PCR: optimization and application in diagnostic virology. Clin. Microbiol. Rev. 13, 559-570 (2000).

8. Mackay, I. M., Arden, K. E. \& Nitsche, A. Real-time PCR in virology. Nucleic Acids Res. 30, 1292-1305 (2002).

9. Gan, S. \& Patel, K. Enzyme immunoassay and enzyme-linked immunosorbent assay. J. Invest. Dermatol. 133, 1-3 (2013).

10. Dobrovolskaia, E., Gam, A. \& Slater, J. E. Competition enzyme-linked immunosorbant assay (ELISA) can be a sensitive method for the specific detection of small quantities of allergen in a complex mixture. Clin. Exp. Allergy 36, 525-530 (2006).

11. Mariani, M. et al. A competitive enzyme-linked immunosorbent assay for measuring the levels of serum antibody to Haemophilus influenzae type b. Clin. Diagn. Lab. Immunol. 5, 667-674 (1998).

12. Faye, O. et al. Quantitative real-time PCR detection of Zika virus and evaluation with field-caught mosquitoes. Virol. J. 10, 1-8 (2013).

13. Fukushima, K. et al. Plaque formation assay for human parainfluenza virus type 1 . Biol. Pharm. Bull. 34, 996-1000 (2011).

14. Pereira, C., Clarke, E. \& Damen, J. Hematopoietic colony-forming cell assays. Methods Mol. Biol. 407, 177-208 (2007).

15. Roingeard, P. Viral detection by electron microscopy: past, present and future. Biol. Cell. 100, 491-501 (2008).

16. Uetrecht, C. et al. Interrogating viral capsid assembly with ion mobility-mass spectrometry. Nature Chem. 3, 126-132 (2011).

17. Sze, S. M. \& Ng, K. (ed. 3) Physics of Semiconductor Devices. (John Wiley \& Sons, Inc., Hoboken, New Jersey, 2007)

18. Lodish, H. et al. Molecular Cell Biology (ed 4.). (W. H. Freeman, New York, 2000).

19. Michen, B. \& Graule, T. Isoelectric points of viruses B. J Applied Micb. 109, 388-397 (2010).
20. Gentile, M. et al. Determination of the size of HIV using adenovirus type 2 as an internal length marker. J Virol Methods. 48, 43-52 (1994).

21. Browning, M., Schmidt, R., Lew, K. \& Rizvi, T. A. Primate and feline lentivirus vector RNA packaging and propagation by heterologous lentivirus virions. J. Virol. 75, 5129-5140 (2001).

22. Rizvi, T. A. et al. Optimal packaging of FIV genomic RNA depends upon a conserved long-range interaction and a palindromic sequence within gag. J. Mol. Biol. 403, 103-119 (2010).

23. Coffin, J. M., Hughes, S. H. \& Varmus, H. E. (eds,) Retroviruses. (Cold Spring Harbor Laboratory Press, New York, 1997).

\section{Acknowledgments}

This work was supported by funds from the UAE National Research Foundation and the Ministry of Higher Education and Research.

\section{Author contributions}

M.A. conceived the concept and performed the electrical experimental work and wrote the manuscript with relevant sections added by F.M. and T.A.R. M.A. and T.A.R. supervised the project. L.M.A. made the virus preparations. F.M. performed the viral quantitation assays and edited the manuscript. All authors discussed the results and commented on the manuscript.

\section{Additional information}

Competing financial interests: The authors declare no competing financial interests.

How to cite this article: Ahmad, M.A., Mustafa, F., Ali, L.M. \& Rizvi, T.A. Virus detection and quantification using electrical parameters. Sci. Rep. 4, 6831; DOI:10.1038/srep06831 (2014)

This work is licensed under a Creative Commons Attribution-NonCommercialNoDerivs 4.0 International License. The images or other third party material in this article are included in the article's Creative Commons license, unless indicated otherwise in the credit line; if the material is not included under the Creative Commons license, users will need to obtain permission from the license holder in order to reproduce the material. To view a copy of this license, visit http:// creativecommons.org/licenses/by-nc-nd/4.0/ 